Research Article

\title{
Fuzzy Adaptive Control for a Class of Nonlinear System with Prescribed Performance and Unknown Dead-Zone Inputs
}

\author{
Chunzhi Yang, ${ }^{1}$ Song $\mathrm{Xu}\left(\mathbb{D}^{2}{ }^{2}\right.$ and Hui $\mathrm{Lv}^{1}$ \\ ${ }^{1}$ School of Finance and Mathematics, Huainan Normal University, Huainan 232038, China \\ ${ }^{2}$ Institute of Urban Economy and Regional Development, Anhui Jianzhu University, Hefei 230009, China \\ Correspondence should be addressed to Song Xu; songxuxs@gmail.com
}

Received 6 September 2020; Revised 11 November 2020; Accepted 30 November 2020; Published 23 December 2020

Academic Editor: Ahmed Mostafa Khalil

Copyright (C) 2020 Chunzhi Yang et al. This is an open access article distributed under the Creative Commons Attribution License, which permits unrestricted use, distribution, and reproduction in any medium, provided the original work is properly cited.

Based on fuzzy logic systems and prescribed performance technology, this paper investigated the stable tracking problem of a class of nonlinear systems with unknown dead-zone inputs. Firstly, the original system is transformed into an equivalent system. Then, a Nussbaum-type function is added in the predefined performance controller to estimate the unknown control direction, and the boundedness of all signals in the closed-loop system is guaranteed. In addition, tracking errors will always remain within the prescribed performance boundaries. Finally, a comparative simulation example shows the effectiveness of the method proposed in this paper.

\section{Introduction}

As is known to all, the dead zone may badly limit the system performance, such as electronic circuits and electric servomotors [1-5]. So, many researchers used different control methods (i.e., backstepping technique, fuzzy systems, neural networks, sliding mode control, and so on) to research the stability of nonlinear systems with unknown control directions [6-9]. For example, based on the terminal sliding mode control, Chen and Ren in [6] proposed an adaptive neural control scheme for permanent magnet synchronous motor (PMSM) servo system with dead-zone input. For uncertain discrete-time systems with nonaffine dead-zone input, Liu and Tong [7] used adaptive neural networks tracking control such that the tracking error converges to a small neighborhood of zero. By using high-dimensional integral Lyapunov-Krasovskii functional theory and direct adaptive control scheme, control of uncertain MIMO systems with time-varying delay and dead-zone input was considered in [8]. In [9], the authors investigated an adaptive backstepping control method for an SISO system with unknown dead-zone input. For eliminating the influence of unknown control directions, one of the commonly applied methods is the Nussbaum-type function technique [10-12].
Liu et al. [10] constructed an adaptive neural controller for discrete-time system, which made all signals of the closedloop system bounded in the condition of unknown deadzone input. For a class of MIMO stochastic systems in [11], $\mathrm{Li}$ and Tong put forward an adaptive fuzzy output-feedback control method by using Nussbaum gain function and fuzzy logic systems. Xu [12] applied the adaptive neural control scheme to guarantee that the flexible hypersonic flight vehicle system is bounded stable with dead-zone input.

However, control methods in aforementioned literature can only guarantee that the tracking error converges to a small residual set, rather than a small residual set with the prescribed performance bounds. For an uncertain nonlinear system $[4,13-15]$, in order to solve the above problem, the prescribed performance control (PPC) strategy has been proposed. The idea of this method is converting the original system to an equivalent system and ensuring the boundedness of the states of the equivalent system. For example, based on the backstepping technique and the PPC, Wang and $\mathrm{Hu}$ [13] proposed an improved PPC for the longitudinal model of an air-breathing hypersonic vehicle (AHV) with unknown dead-zone input nonlinearity. Yang and Chen [14] developed an adaptive neural PPC for near space vehicles (NSVs) with input nonlinearities including saturation and 
dead zone. It can be seen from the literature $[13,14]$ that it has certain practical significance to adopt the PPC method in the practical system. For MIMO nonlinear systems with unknown dead-zone inputs, Shi et al. [4] proposed an adaptive fuzzy PPC scheme such that only three parametric adaptive rules were needed. Cao et al. [16] studied a fractional neural network model with impulse amplitude, timedelay time-varying, and reaction diffusion terms. Many other interesting results about PPC of nonlinear systems can be seen in [17-19].

Inspired by the work of $[4,20]$, this paper explores the stability problem that the tracking error meets prescribed performance boundary ( $\mathrm{PPB}$ ) with unknown dead-zone input. Compared with related works, the main contributions of this paper are listed as follows: (1) compared with the work of $[14,17,19]$, the introduced error transformation variable $z_{i}$ can be directly differentiated without the participation of the transformation function. (2) The Nussbaum-type function is incorporated into the control method so that the influence of unknown control directions is eliminated. (3) The singular problem in the PPC for nonlinear systems can be avoided by using the proposed method.

The structure of this paper is as follows. In Section 2, the uncertain system model with unknown dead-zone inputs is raised and provides assumption and lemmas. Section 3 includes the designation of fuzzy adaptive prescribed performance control method. The results of simulation are presented in Section 4. In Section 5, a brief conclusion is given.

\section{Preliminaries}

Consider the following uncertain nonlinear system, which described as

$$
\left\{\begin{array}{l}
\dot{x}_{1}=f_{1}(t, x)+d_{1}(t)+b_{1} \phi_{1}\left(u_{1}\right), \\
\dot{x}_{2}=f_{2}(t, x)+d_{2}(t)+b_{2} \phi_{2}\left(u_{2}\right), \\
\cdots, \\
\dot{x}_{n}=f_{n}(t, x)+d_{n}(t)+b_{n} \phi_{n}\left(u_{n}\right),
\end{array}\right.
$$

where $x=\left[x_{1}, x_{2}, \ldots, x_{n}\right]^{T}$ is the system state vector, $f_{i}(t, x)$ is an unknown smooth nonlinear function, $d_{i}(t)$ is an external disturbance, $\phi_{i}\left(u_{i}\right)$ denotes the control input subject to dead-zone type nonlinear, and $b_{i}$ is an unknown control coefficient, $i=1,2, \ldots, n$. Here, $\phi\left(u_{i}\right)$ is described as follows:

$$
\phi_{i}\left(u_{i}\right)=\left\{\begin{array}{l}
m_{i}\left(u_{i}-b_{i r}\right), \quad \text { if } u_{i} \geq b_{i r} \\
0, \quad \text { if } b_{i l}<u_{i}<b_{i r} \\
m_{i}\left(u_{i}+b_{i l}\right), \quad \text { if } u_{i} \leq-b_{i l},
\end{array}\right.
$$

where $m_{i}, b_{i r}$, and $b_{i l}$ are unknown positive constants, $i=1,2, \ldots, n$. Then, we rewrite (2) as

$$
\phi_{i}\left(u_{i}\right)=m_{i} u_{i}+\triangle u_{i}
$$

where

$$
\Delta u_{i}= \begin{cases}-m_{i} b_{i r}, & \text { if } u_{i} \geq b_{i r} \\ -m_{i} u_{i}, & \text { if } b_{i l}<u_{i}<b_{i r} \\ m_{i} b_{i l}, & \text { if } u_{i} \leq-b_{i l} .\end{cases}
$$

From (3), system (1) is described as

$$
\left\{\begin{array}{l}
\dot{x}_{1}=f_{1}(t, x)+d_{1}(t)+b_{1} m_{1} u_{1}+b_{1} \Delta u_{1}, \\
\dot{x}_{2}=f_{2}(t, x)+d_{2}(t)+b_{2} m_{2} u_{2}+b_{2} \Delta u_{2}, \\
\ldots, \\
\dot{x}_{n}=f_{n}(t, x)+d_{n}(t)+b_{n} m_{n} u_{n}+b_{n} \Delta u_{n} .
\end{array}\right.
$$

Designing two adaptive prescribed performance control methods is the control objective of this paper. The goal of this paper is that the tracking error $e_{i}$ satisfies PPB, and all closed-loop system signals are bounded.

In order to meet the objective, we proposed the following assumptions.

Assumption 1. The system state $x$, the reference signal $x_{d}$, and its derivative $\dot{x}_{d}$ are available and bounded.

Assumption 2. The nonlinear function $f_{i}(t, x)$, the control coefficient $b_{i}$, and the external disturbance $d_{i}(t)$ are unknown but bounded, $i=1,2, \ldots, n$.

Remark 1. Assumptions 1 and 2 are commonly used to address the problem of unknown control direction [21-23]. And owing to the boundedness of $b_{i}, \Delta u_{i}$, and $d_{i}(t)$, we know that there exists an unknown positive constant $D_{i}^{*}$, such that $\left|b_{i} \Delta u_{i}+d_{i}(t)\right| \leq D_{i}^{*}, i=1,2, \ldots, n$.

Let $\bar{b}_{i}=b_{i} m_{i}$ and $D_{i}(t)=b_{i} \Delta u_{i}+d_{i}(t)$, then $\left|D_{i}(t)\right| \leq$ $D_{i}^{*}$. The error state is defined as $e=\left[e_{1}, e_{2}, \ldots\right.$, $\left.e_{n}\right]^{T}=\left[x_{1}-x_{1 d}, x_{2}-x_{2 d}, \ldots, x_{n}-x_{n d}\right]^{T}$, and the following error system is acquired:

$$
\left\{\begin{array}{l}
\dot{e}_{1}=f_{1}(t, x)+D_{1}(t)+\bar{b}_{1} u_{1}(t)-\dot{x}_{1 d} \\
\dot{e}_{2}=f_{2}(t, x)+D_{2}(t)+\bar{b}_{2} u_{2}(t)-\dot{x}_{2 d} \\
\cdots \\
\dot{e}_{n}=f_{n}(t, x)+D_{n}(t)+\bar{b}_{n} u_{n}(t)-\dot{x}_{n d}
\end{array}\right.
$$

The following definition and lemmas used in this paper are to overcome the problem of unknown control directions.

Definition 1. A continuous function $N(\cdot)$ is called a Nussbaum function, if $N(\cdot)$ satisfies

$$
\begin{aligned}
& \lim _{t \rightarrow+\infty} \sup \frac{1}{t} \int_{0}^{t} N(s) \mathrm{d} s=+\infty \\
& \lim _{t \rightarrow+\infty} \inf \frac{1}{t} \int_{0}^{t} N(s) \mathrm{d} s=-\infty
\end{aligned}
$$

Remark 2. Generally, the selection of the Nussbaum functions for the controller design with unknown control direction is $\xi^{2} \cos (\xi)$ or $e^{\xi^{2}} \cos ((\Pi / 2) \xi)$ or $\ln (\xi+1)$ 
$\cos (\sqrt[2]{\ln (\xi+1)})$ and so on. In this paper, we choose the even Nussbaum function $\xi^{2} \cos (\xi)$ to design the PPC controller.

Lemma 1 (see [15]). Let $V(t)$ and $\xi(t)$ be smooth functions, $V(t) \geq 0$ for $\forall t \in\left[0, t_{f}\right)$, and $N(\cdot)$ be an even smooth Nussbaum function. If the following inequality holds:

$$
\begin{array}{r}
V(t) \leq r_{0}+e^{-r_{1} t} \int_{0}^{t}(\alpha(x(s)) N(\xi(s))+1) \cdot \dot{\xi}(s) e^{-r_{1} s} \mathrm{~d} s, \\
\forall t \in\left[0, t_{f}\right),
\end{array}
$$

where $r_{0}$ and $r_{1}$ are constants with $r_{1}>0, \alpha(x(t))$ is a timevarying parameter which takes values in the unknown closed intervals $I=:\left[l^{-}, l^{+}\right]$with $0 \bar{\epsilon} I$, then $V(t), \xi(t)$, and $\int_{0}^{t}(\alpha(x(s)) N(\xi(s))+1) \dot{\xi}(s) e^{-r_{1} s} d s$ must be bounded on $\left[0, t_{f}\right)$.

Lemma 2 (see [4]). Let $A \in R^{n \times n}$ be a symmetric matrix and $x \in R^{n}$ be a nonzero vector. Let $\alpha=\left(x^{T} A x / x^{T} x\right)$. Then, there exist eigenvalues of $A$ such that $\lambda_{1} \in(-\infty, \alpha]$ and $\lambda_{2} \in[\alpha,+\infty)$.

Remark 3. In this paper, we will construct the time-varying parameter $\alpha(t)$ by using Lemma 2. For example, let $A=$ $\operatorname{diag}\left(a_{1}, a_{2}, \ldots, a_{n}\right)$ and $z$ is a nonzero vector; we can design $\alpha(t)$ as $\alpha(t)=\left(z^{T} A z / z^{T} z\right)$, and then there exist two constants $l^{-}$and $l^{+}$such that

$$
l^{-} \leq \min _{i}\left\{a_{i}\right\} \leq \alpha(t) \leq \max _{i}\left\{a_{i}\right\} \leq l^{+} .
$$

In this paper, fuzzy logic systems will be used to approximate unknown nonlinear functions. Generally, the output of fuzzy logic system can be expressed as [24-32]

$$
\widehat{f}=\frac{\sum_{i \in I} w_{i}(t) \mu_{i}(x(t))}{\sum_{i \in I} \mu_{i}(x(t))},
$$

where $x=\left[x_{1}(t), \ldots, x_{n}(t)\right]^{T} \in C^{1}[\mathscr{F}, \Omega](\forall t \in \mathscr{F})$ is the input vector. $\widehat{f}: \Omega \mapsto \mathscr{R}$ is a Lipschitz continuous mapping, $\Omega \subseteq \mathscr{R}^{n}$ is a compact subset, and $\mathscr{R}$ is a real line. $I=\prod_{j=1}^{n} \mathscr{F}_{j}$, where $\mathscr{F}_{j}$ consists of $N_{j}$ fuzzy sets $(1 \leq j \leq n)$. The membership function of the $i$-th rule is $\mu_{i}(i \in I)$, where $\mu_{i}: \mathscr{R}^{n} \mapsto[0,1] . w_{i}: \mathscr{I} \mapsto \mathscr{R}(i \in 1,2, \ldots, N)$, which represents the centroid of the $i$-th consequent set. Let $\theta(t)=$ $\left[w_{1}(t), \ldots, w_{N}(t)\right]^{T} \quad$ and $\quad \varphi(t)=\left[\varphi_{1}(x(t)), \varphi_{2}(x(t))\right.$, $\left.\ldots, \varphi_{N}(x(t))\right]^{T}$, where $\varphi_{i}(i \in I)$ is the $i$-th fuzzy basis function and $\varphi_{i}$ is a continuous mapping, then $\varphi_{i}$ can be defined as

$$
\varphi_{i}(x(t))=\frac{\mu_{i}(t)}{\sum_{s \in I} \mu_{s}(x(t))} .
$$

Thus, (10) can be rearranged as

$$
\widehat{f}(x(t))=\theta^{T}(t) \varphi(t) .
$$

\section{Synchronization Controller Design}

3.1. Prescribed Performance. The steady-state performance of $e_{i}$ can be preserved by setting the performance constraint condition as

$$
-\lambda_{2} \mu(t)<e_{i}<\lambda_{1} \mu(t), \quad i=1,2, \ldots, n,
$$

where $\lambda_{1}$ and $\lambda_{2}$ are positive constants, which can regulate the error boundaries. $\mu(t)$ is denoted by

$$
\mu(t)=\left(\mu_{0}-\mu_{\infty}\right) e^{-\bar{\lambda} t}+\mu_{\infty},
$$

where $\bar{\lambda}>0$ and $\mu_{0}, \mu_{\infty}>0$. Obviously, $\mu(t)$ is a decreasing function and $\mu(0)=\mu_{0}, \lim _{t \rightarrow+\infty} \mu(t)=\mu_{\infty}$.

We abbreviate $\mu(t)$ to $\mu$ and give an error transformation as

$$
z_{i}=\frac{\lambda_{1} e_{i}}{\lambda_{1} \mu-e_{i}} l\left(e_{i}\right)+\frac{\lambda_{2} e_{i}}{\lambda_{2} \mu+e_{i}}\left(1-l\left(e_{i}\right)\right),
$$

where $l\left(e_{i}\right)$ satisfies

$$
l\left(e_{i}\right)= \begin{cases}1, & e_{i} \geq 0 \\ 0, & e_{i}<0 .\end{cases}
$$

Obviously, if we choose the initial condition $e_{i}(0)$ as $-\lambda_{2} \mu(0)<e_{i}(0)<\lambda_{1} \mu(0)$ and $z_{i}$ is bounded, then the error state $e_{i}$ will satisfy the inequality that $-\lambda_{2} \mu<e_{i}<\lambda_{1} \mu$. Because when $e_{i}$ approaches $-\lambda_{2} \mu(0)$ or $\lambda_{1} \mu(0)$, $z_{i}$ will approach infinity, which contradicts the boundedness of $z_{i}$. So, here, the work for us is to prove that the error transformation variable $z_{i}$ is bounded, $z_{i} i=1,2, \ldots, n$.

Remark 4. The proposed transformation variable $z_{i}$ in this paper can be directly expressed by $e_{i}$ and parameters $\lambda_{1}$ and $\lambda_{2}$, see (15), while the traditional transformation variable $z_{i}$ in $[4,14]$ needs to rely on the transformation function $\tanh \left(z_{i}\right)=\left(e_{i} / \mu\right)$ to transform. Therefore, the proposed error transformation variable $z_{i}$ is simpler and is more convenient for the design of the controller $u_{i}$.

3.2. Control Design and Stability Analysis. The time derivative of $z_{i}$ is

$$
\dot{z}_{i}=\mu \Pi_{i} \dot{e}_{i}-\dot{\mu} \Pi_{i} e_{i},
$$

where $\Pi_{i}=\left(\lambda_{1}^{2} /\left(\lambda_{1} \mu-e_{i}\right)^{2}\right) l\left(e_{i}\right)+\left(\lambda_{2}^{2} /\left(\lambda_{2} \mu+e_{i}\right)^{2}\right)\left(1-l\left(e_{i}\right)\right)$. So, we obtain the following error transformation dynamic system.

Due to the fact that $f_{i}(t, x)$ is unknown, we need to employ fuzzy logic system to approximate $f_{i}(t, x)$. So, the approximate of function $f_{i}(t, x)$ can be expressed as $\widehat{f}_{i}\left(x, \widehat{\theta}_{f_{i}}\right)=\widehat{\theta}_{f_{i}}^{T} \varphi_{f_{i}}(x), i=1,2, \ldots, n$, where $\hat{\theta}_{f_{i}}$ is the parameter vector and the fuzzy function vector is $\varphi_{f_{i}}(x)$. For $f_{i}(t, x)$, there is an optimal parameter vector $\theta_{f_{i}}^{*}$ such that

$$
f_{i}(t, x)=\theta_{f_{i}}^{* T} \varphi_{f_{i}}(x)+\varepsilon_{f_{i}}(x),
$$


where $\quad \theta_{f_{i}}^{*}=\arg \min _{\hat{\theta}_{f_{i}}}\left\{\sup \left|f_{i}(t, x)-\hat{\theta}_{f_{i}}^{T} \varphi_{f_{i}}(x)\right|\right\} \quad$ and $\varepsilon_{f_{i}}(x)$ is the approximation error, which is bounded as $\left|\varepsilon_{f_{i}}(x)\right| \leq \varepsilon_{f_{i}}^{*}$, where $\varepsilon_{f_{i}}^{*}$ is a positive constant, $i=1,2, \ldots, n$.
Let $\bar{D}_{i}(t)=\varepsilon_{f_{i}}(x)+D_{i}(t)$, then $|\bar{D}(t)| \leq \varepsilon_{f_{i}}^{*}+D_{i}^{*} \triangleq \bar{D}_{i}^{*}$. The error transformation dynamic system is rewritten as follows:

$$
\left\{\begin{array}{l}
\dot{z}_{1}=\mu \Pi_{1}\left(f_{1}(t, x)+D_{1}(t)+\bar{b}_{1} u_{1}(t)-\dot{x}_{1 d}\right)-\dot{\mu} \Pi_{1} e_{1}, \\
\dot{z}_{2}=\mu \Pi_{2}\left(f_{2}(t, x)+D_{2}(t)+\bar{b}_{2} u_{2}(t)-\dot{x}_{2 d}\right)-\dot{\mu} \Pi_{2} e_{2}, \\
\cdots \\
\left.\dot{z}_{n}=\mu \Pi_{n} f_{n}(t, x)+D_{n}(t)+\bar{b}_{n} u_{n}(t)-\dot{x}_{n d}\right)-\dot{\mu} \Pi_{n} e_{n} .
\end{array}\right.
$$

Next, we design the controller $u_{i}$ to guarantee the boundedness of $z_{i}$. Let $z=\left[z_{1}, z_{2}, \ldots, z_{n}\right]^{T}, \Pi=\left[\Pi_{1}, \Pi_{2}\right.$, $\left.\ldots, \Pi_{n}\right]^{T}$, and another controller as follows:

$$
\begin{aligned}
& u_{i}=\frac{z_{i} N(\xi) \bar{u}}{\mu \Pi_{i}}, \\
& \bar{u}=\frac{\Delta^{2}}{\Delta\|z\|+\sigma}, \\
& \dot{\xi}=\|z\|^{2} \bar{u},
\end{aligned}
$$

where $\Delta=\Delta_{1}+\Delta_{2}, \Delta_{1}=\mu \hat{\theta}\|\Pi\| \sqrt{\sum_{i=1}^{n}\left\|\varphi_{f_{i}}(x)\right\|^{2}}, \Delta_{2}=\mu\|\Pi\|$ $\widehat{D}+\sum_{i=1}^{n}|\dot{\mu}| \Pi_{i}\left|e_{i}\right|+(1 / 2)\|z\|, \quad \theta^{*} \triangleq \sqrt{\sum_{i=1}^{n}\left\|\theta_{f_{i}}^{*}\right\|^{2}}, \quad D^{*}=\sum_{i=1}^{n}$
$\bar{D}_{i}^{*}, \widehat{\theta}$ and $\widehat{D}$ are the estimates of $\theta^{*}$ and $D^{*}$, respectively, and the parameters adaptive laws are given as

$$
\dot{\hat{\theta}}=\mu\|\Pi\| \sqrt{\sum_{i=1}^{n}\left\|\varphi_{f_{i}}(x)\right\|^{2}} \cdot\|z\|-\gamma_{1} \hat{\theta}, \dot{\hat{D}}=\mu\|\Pi\|\|z\|-\gamma_{2} \widehat{D},
$$

where $\gamma_{1}$ and $\gamma_{2}$ are positive constants.

Now, consider the Lyapunov function:

$$
V=\frac{1}{2} z^{T} z+\frac{1}{2} \widetilde{\theta}^{2}+\frac{1}{2} \widetilde{D}^{2},
$$

where $\widetilde{\theta}=\theta^{*}-\widehat{\theta}$ and $\widetilde{D}=D^{*}-\widehat{D}$. By differentiating $V$, one achieves

$$
\begin{aligned}
\dot{V}= & -\frac{1}{2} z^{T} z+z^{T}\left(\dot{z}+\frac{z}{2}\right)+\tilde{\theta} \dot{\tilde{\theta}}+\widetilde{D} \dot{\widetilde{D}} \\
= & -\frac{1}{2} z^{T} z+\sum_{i=1}^{n}\left(z_{i} \mu \Pi_{i} \theta_{f_{i}}^{* T} \varphi_{f_{i}}(x)+z_{i} \mu \Pi_{i} \bar{D}_{i}(t)-\dot{\mu} z_{i} \Pi_{i} e_{i}-z_{i} \dot{x}_{i d} \mu \Pi_{i}+\frac{z_{i}^{2}}{2}\right)+\sum_{i=1}^{n} z_{i} \mu \Pi_{i} \bar{b}_{i} u_{i}+\tilde{\theta} \dot{\tilde{\theta}}+\widetilde{D} \dot{\tilde{D}} \\
\leq & -\frac{1}{2} z^{T} z+\sum_{i=1}^{n}\left(\left|z_{i}\right| \mu \Pi_{i}\left\|\theta_{f_{i}}^{*}\right\|\left\|\varphi_{f_{i}}(x)\right\|+\left|z_{i}\right| \mu \Pi_{i}\left|\bar{D}_{i}(t)\right|+|\dot{\mu}|\left|z_{i}\right| \Pi_{i}\left|e_{i}\right|+\left|z_{i}\right|\left|\dot{x}_{i d}\right| \mu \Pi_{i}+\frac{z_{i}^{2}}{2}\right)+\sum_{i=1}^{n} z_{i} \mu \Pi_{i} \bar{b}_{i} u_{i}+\tilde{\theta} \dot{\tilde{\theta}}+\widetilde{D} \dot{\tilde{D}} \\
\leq & -\frac{1}{2} z^{T} z+\mu\|z\|\|\Pi\| \cdot \sum_{i=1}^{n}\left\|\theta_{f_{i}}^{*}\right\||| \varphi_{f_{i}}(x)\|+\| z\|\mu\| \Pi\left\|\sum_{\sum_{i=1}^{n} D_{i}^{*}=\widetilde{D}+\hat{D}}^{*}+\right\| z\left\|\cdot \sum_{i=1}^{n}|\dot{\mu}| \Pi_{i}\left|e_{i}\right|+\right\| z\left\|\sum_{i=1}^{n}\left|\dot{x}_{i d}\right| \mu \Pi_{i}+\frac{1}{2}\right\| z \|^{2} \\
& +\sum_{i=1}^{n} z_{i} \mu \Pi_{i} \bar{b}_{i} u_{i}+\tilde{\theta} \dot{\tilde{\theta}}+\widetilde{D} \dot{\tilde{D}} .
\end{aligned}
$$


According to Cauchy-Schwarz inequality,

$$
\begin{aligned}
\sum_{i=1}^{n}\left\|\theta_{f_{i}}^{*}\right\|\left\|\varphi_{f_{i}}(x)\right\| & \leq \sqrt{\sum_{i=1}^{n}\left\|\theta_{f_{i}}^{*}\right\|^{2}} \cdot \sqrt{\sum_{i=1}^{n}\left\|\varphi_{f_{i}}(x)\right\|^{2}} \\
& =\theta^{*} \sqrt{\sum_{i=1}^{n}\left\|\varphi_{f_{i}}(x)\right\|^{2}} \\
& =(\tilde{\theta}+\widehat{\theta}) \sqrt{\sum_{i=1}^{n}\left\|\varphi_{f_{i}}(x)\right\|^{2}} .
\end{aligned}
$$

Considering its adaptive law (21), it is concluded that

$$
\begin{aligned}
\tilde{\theta} \dot{\tilde{\theta}} & =-\tilde{\theta} \dot{\tilde{\theta}} \\
& =-\tilde{\theta}\left(\mu\|\Pi\| \sqrt{\sum_{i=1}^{n}\left\|\varphi_{f_{i}}(x)\right\|^{2}} \cdot\|z\|-\gamma_{1} \widehat{\theta}\right) \\
& =-\tilde{\theta} \mu\|\Pi\| \sqrt{\sum_{i=1}^{n}\left\|\varphi_{f_{i}}(x)\right\|^{2}} \cdot\|z\|+\gamma_{1} \tilde{\theta} \widehat{\theta} \\
& =-\tilde{\theta} \mu\|\Pi\| \sqrt{\sum_{i=1}^{n}\left\|\varphi_{f_{i}}(x)\right\|^{2} \cdot\|z\|+\gamma_{1} \tilde{\theta}\left(\theta^{*}-\tilde{\theta}\right)} \\
& \leq-\frac{\gamma_{1}}{2} \tilde{\theta}^{2}-\tilde{\theta} \mu\|\Pi\| \sqrt{\sum_{i=1}^{n}\left\|\varphi_{f_{i}}(x)\right\|^{2}} \cdot\|z\|+\frac{\gamma_{1}}{2} \theta^{* 2} .
\end{aligned}
$$

Similarly,

$$
\widetilde{D} \dot{\widetilde{D}} \leq-\frac{\gamma_{2}}{2} \widetilde{D}^{2}-\widetilde{D} \mu\|\Pi\|\|z\|+\frac{\gamma_{2}}{2} D^{* 2} .
$$

So, one gets

$$
\begin{aligned}
\dot{V} \leq & -\overline{\bar{\gamma}} V+\|z\|\left(\mu\|\Pi\| \widehat{\theta} \sum_{i=1}^{n} \sqrt{\left\|\varphi_{f_{i}}(x)\right\|^{2}}\right) \\
& +\mu\|\Pi\| \widehat{D}+\sum_{i=1}^{n}|\dot{\mu}| \Pi_{i}\left|e_{i}\right|+\sum_{i=1}^{n}\left|\dot{x}_{i d}\right| \mu \Pi_{i} \\
& \left.+\frac{\|z\|}{2}\right)+\sum_{i=1}^{n} z_{i} \bar{b} z_{i} N(\xi) \bar{u}+\overline{\bar{R}}_{0},
\end{aligned}
$$

where $\overline{\bar{\gamma}}=\min \left\{1, \gamma_{1}, \gamma_{2}\right\}$ and $\overline{\bar{R}}_{0}=\left(\gamma_{1} / 2\right) \theta^{* 2}+\left(\gamma_{2} / 2\right) D^{* 2}$. For $z_{i} \neq 0$, we define $\alpha(t)=\left(\left(\sum_{i=1}^{n} z_{i} \bar{b}_{i} z_{i}\right) /\left(\sum_{i=1}^{n} z_{i}^{2}\right)\right)=$ $\left(z^{T} U z / z^{T} z\right)$, where $U=\operatorname{diag}\left(\bar{b}_{1}, \bar{b}_{2}, \ldots, \bar{b}_{n}\right)$. By using Lemma 2 , there are two constants $l^{+}$and $l^{-}$such that $l^{-} \leq \min _{i} \bar{b}_{i} \leq \alpha(t) \leq \max _{i} b_{i} \leq l^{+}$. So,

$$
\sum_{i=1}^{n} z_{i} \bar{b} z_{i}=\|z\|^{2} \alpha(t)
$$

Obviously, if $z_{i}=0,(28)$ also holds. By substituting $u_{i}(t)$ in (20) into (27), it yields

$$
\dot{V} \leq-\overline{\bar{\gamma}} V+(\alpha(t) N(\xi)+1) \dot{\xi}+\overline{\bar{R}}_{0},
$$

where $\overline{\bar{R}}_{0}=\overline{\bar{R}}_{0}+\sigma$. Integrating (29) over $[0, t]$, one obtains

$$
V \leq V(0)+\frac{\overline{\bar{R}}_{0}}{\overline{\bar{\gamma}}}+\int_{0}^{t}(1+\alpha(s) N(\xi)) \dot{\xi} e^{\overline{\bar{\gamma}}(s-t)} \mathrm{d} s .
$$

According to Lemma 2, $V(t)$ is bounded into $\left[0, t_{f}\right)$. So, $z_{i}$ is bounded for any given positive constant $t_{f}$, and then the boundedness of $z_{i}$ is true for $t_{f}=+\infty$ (see [20]).

Through the above analysis and discussion, the following theorem is obtained.

Theorem 1. Consider the uncertain nonlinear system (1) with dead-zone inputs and unknown control directions. The proposed control method (20) can guarantee the boundedness of all the signals in the closed-loop system. Furthermore, for any $e_{i}(0)$ satisfies $-\lambda_{2} \mu(0)<e_{i}(0)<\lambda_{1} \mu(0)$, the error $e_{i}$ will satisfy the prescribed performance (13).

\section{Simulation Studies}

Consider the Chen chaotic system as a simulation example:

$$
\left\{\begin{array}{l}
\dot{x}_{1}=\underbrace{35 x_{2}-35 x_{1}}_{f_{1}(x)}+d_{1}(t)+b_{1} \phi_{1}\left(u_{1}\right), \\
\dot{x}_{2}=\underbrace{-x_{1} x_{3}-7 x_{1}+28 x_{2}}_{f_{2}(x)}+d_{2}(t)+b_{2} \phi_{2}\left(u_{2}\right), \\
\dot{x}_{3}=\underbrace{-3 x_{3}+x_{1} x_{2}}_{f_{3}(x)}+d_{3}(t)+b_{3} \phi_{3}\left(u_{3}\right),
\end{array}\right.
$$

where $d_{1}(t)=d_{2}(t)=d_{3}(t)=2 \sin (2 t), b_{1}=2, b_{2}=-2, b_{3}$ $=2$, and $\phi_{i}\left(u_{i}\right)$ is described as

$$
\phi_{i}\left(u_{i}\right)= \begin{cases}3\left(u_{i}-2\right), & u_{i} \geq 2, \\ 0, & -2<u_{i}<2, \\ 3\left(u_{i}+2\right), & u_{i} \leq-2,\end{cases}
$$

$i=1,2,3$. One reference signal $x_{d}=[\sin (t) \sin (t) \sin (t)]^{T}$. Choose the fuzzy membership function as.

Firstly, we use the method in [15] to control Chen chaotic system (31), and controller is designed as follows:

$$
\left\{\begin{array}{l}
u_{i}=\xi_{i}^{2} \cos \left(\xi_{i}\right) \bar{u}_{i}, \\
\bar{u}_{i}=\widehat{\eta}_{f_{i}}^{T} \varphi_{f_{i}}(x)+\hat{\rho} \tanh \left(\frac{e_{i}}{\varepsilon_{i}}\right)+c_{i} e_{i}, \\
\dot{\xi}_{i}=e_{i} \bar{u}_{i}, \\
\dot{\hat{\eta}}_{f_{i}}=e_{i} \varphi_{f_{i}}(x)-\gamma_{1 i} \widehat{\eta}_{f_{i}}, \\
\dot{\hat{\rho}}_{i}=e_{i} \tanh \left(\frac{e_{i}}{\varepsilon}\right)-\gamma_{2 i} \hat{\rho}_{i}, \quad i=1,2,3,
\end{array}\right.
$$




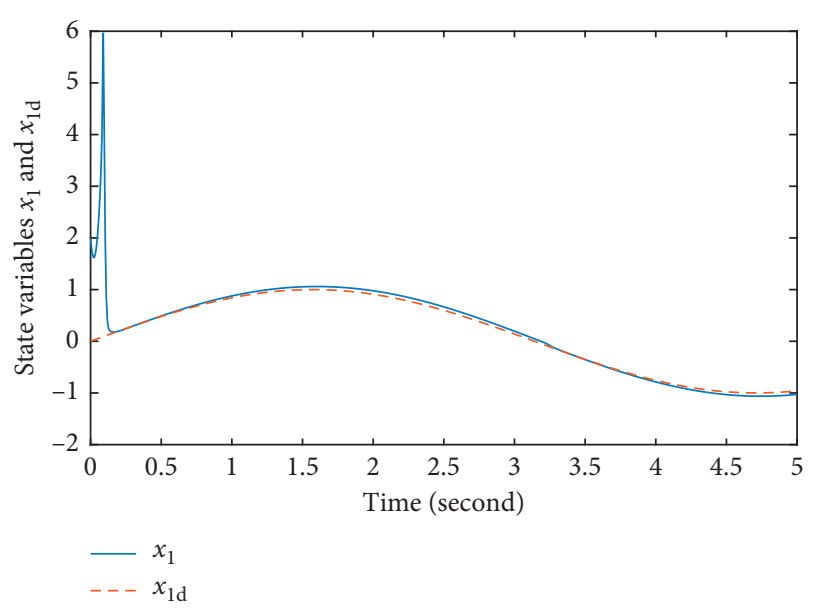

FIgURE 1: $x_{1}$ and $x_{1 d}$ with control scheme (33).

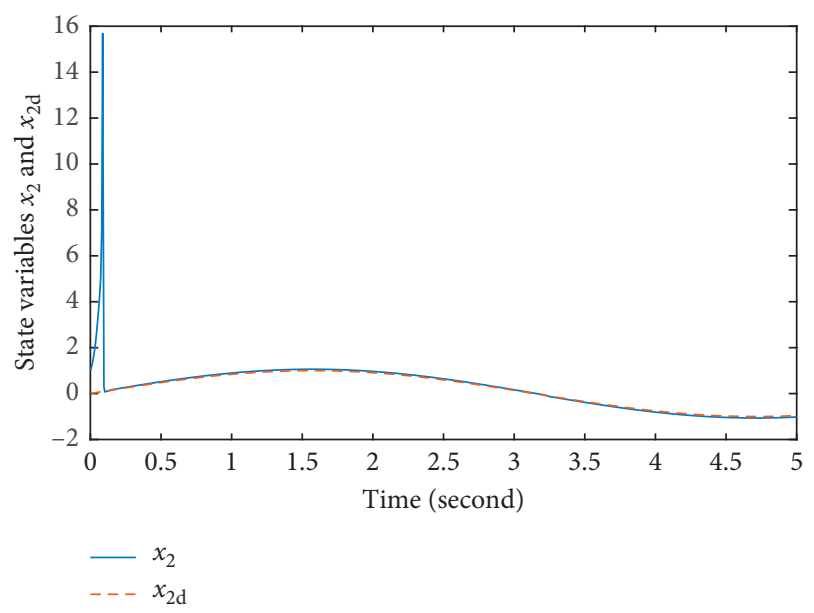

FIgURE 2: $x_{2}$ and $x_{2 d}$ with control scheme (33).

where $\widehat{\eta}_{f_{i}}^{T} \varphi_{f_{i}}(x)$ is the estimate of $f_{i}(x)$ and $f_{i}(x)=\eta$ ${ }_{f_{i}}^{* T} \varphi_{f_{i}}(x)+\varepsilon_{f_{i}}(x)$, where $\varepsilon_{f_{i}}(x)$ is the bounded approximation error. Let $\left|d_{i}(t)+\varepsilon_{f_{i}}(x)+\dot{x}_{i d}\right| \leq \rho_{i}$, where $\widehat{\rho}_{i}$ is the estimate of $\rho_{i}$. The parameters are $\varepsilon=0.5, \gamma_{i 1}=3, \gamma_{i 2}=2$, $i=1,2,3$. The initial values are $x_{1}(0)=2, x_{2}(0)=1, x_{3}(0)=$ $-2, \xi_{1}(0)=0.01, \xi_{2}(0)=0.1, \xi_{3}(0)=0.1, \widehat{\rho}_{1}(0)=0.05, \widehat{\rho}_{2}(0$ )$=-0.3, \hat{\rho}_{3}(0)=0.4, \widehat{\eta}_{f_{1}}(0)=\widehat{\eta}_{f_{2}}(0)=\widehat{\eta}_{f_{3}}(0)=0, \mu(t)=3$ $e^{-2.5 t}+0.1$. By using control scheme (33), the trajectories of $x_{1}, x_{2}$, and $x_{3}$ are shown in Figures 1-3. The corresponding error trajectories are shown in Figure 4. It can be seen that, in the initial control stage, $e_{1}$ and $e_{2}$ will have a jump beyond the prescribed performance boundary (PPB) $[-\mu(t), \mu(t)]$ and then remain within the BBP $[-\mu(t), \mu(t)]$, while $e_{3}$, on the contrary, is within the $\mathrm{PPB}[-\mu(t), \mu(t)]$ at the initial stage and stays outside the PPB $[-\mu(t), \mu(t)]$ after 3 seconds.

In order to compare with the control scheme (33), we employ our proposed control scheme (20). The parameters are $\lambda_{1}=\lambda_{2}=1$ and $\sigma=0.5$, and the initial values are $\widehat{\bar{D}}_{1}=0.2, \hat{\bar{D}}_{2}=0.3$, and $\hat{\bar{D}}_{3}=0.4$, Other parameters and initial values in this example are same as those of the control scheme (33). The simulation results are shown in Figures 5-8. The states $x_{1}, x_{2}$, and $x_{3}$ can track the reference

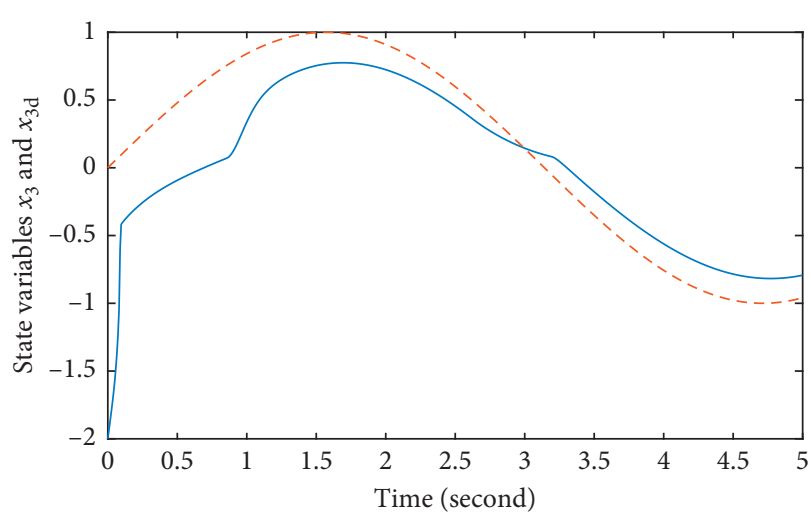

$-x_{3}$

$--x_{3 \mathrm{~d}}$

FIgURE 3: $x_{3}$ and $x_{3 d}$ with control scheme (33).

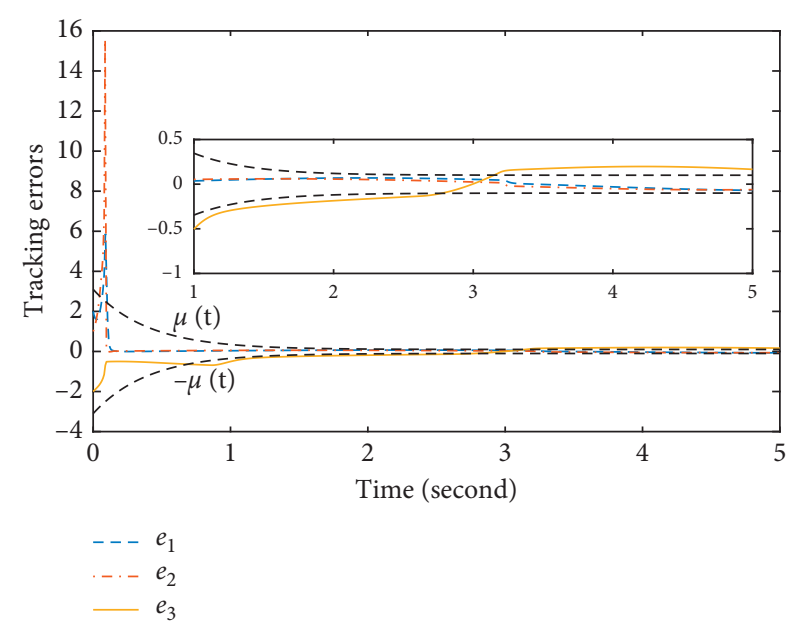

Figure 4: $e_{1}, e_{2}$, and $x_{3}$ with control scheme (33).

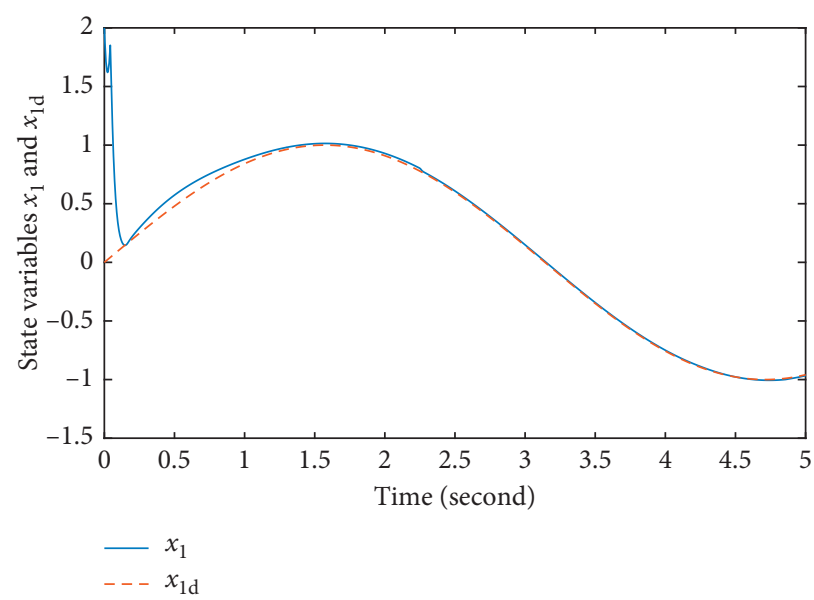

Figure 5: $x_{1}$ and $x_{1 d}$ with proposed control scheme (20).

signal $x_{1 d}, x_{2 d}$, and $x_{3 d}$ quickly after a transient response process in Figures 5-7. From Figure 8, errors $e_{1}, e_{2}$, and $e_{3}$ are not beyond the PPB $[-\mu(t), \mu(t)]$. In Figure 9, two 


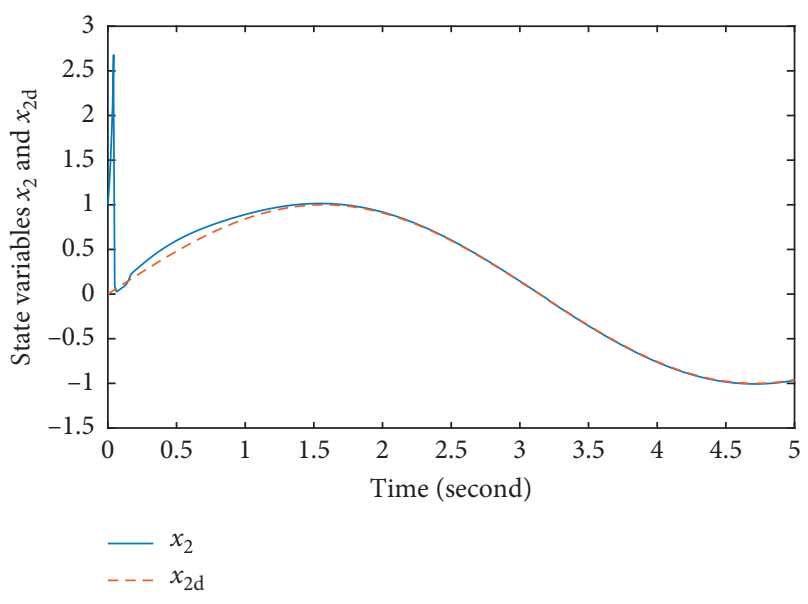

FIGURE 6: $x_{2}$ and $x_{2 d}$ with proposed control scheme (20).

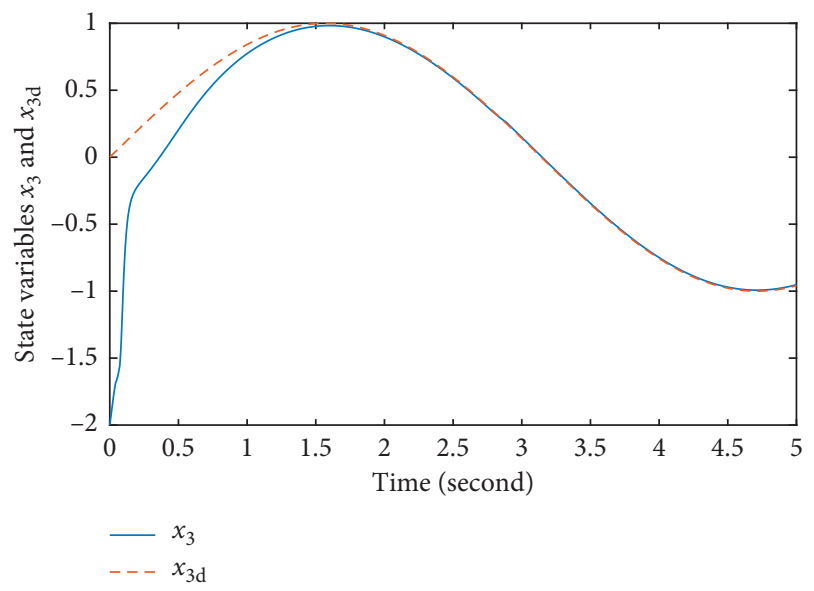

Figure 7: $x_{3}$ and $x_{3 d}$ with proposed control scheme (20).

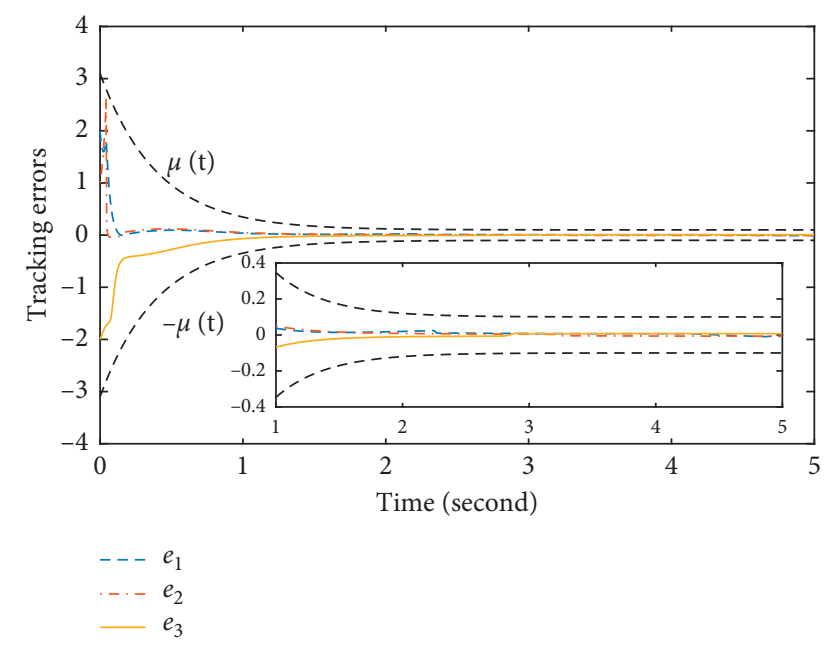

FIgURE 8: $e_{1}, e_{2}$, and $x_{3}$ with proposed control scheme (20).

control effects of error state $\|e\|$ are compared directly. So, these results verify that the proposed control scheme (20) has a better control effect than control scheme (33).

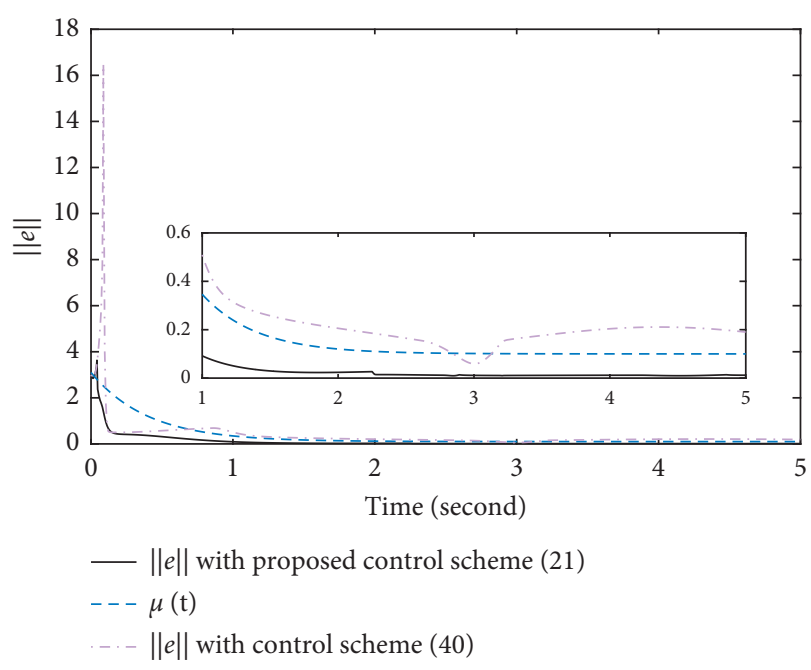

Figure 9: \|e\| with control schemes (20) and (33).

\section{Conclusions}

The problem of robust fuzzy adaptive prescribed performance control for uncertain nonlinear system is investigated. The proposed control approach is to make sure that all signals in closed-loop system are bounded and tracking errors converge to a small neighborhood of zero with the prescribed performance bounds. The Nussbaum-type function is incorporated into two control methods such that the influence of unknown control directions is eliminated. It is unnecessary to use the inverse operation in the proposed methods. Simulation results verify the effectiveness and robustness of the proposed method. How to effectively estimate the unknown function of the nonlinear system is the future research direction.

\section{Data Availability}

All datasets generated for this study are included within the manuscript.

\section{Conflicts of Interest}

The authors declare that they have no conflicts of interest.

\section{Acknowledgments}

This work was supported by the Key Natural Science Research Projects in Anhui Universities (KJ2019A0695), the Natural Science Research Projects of Huainan Normal University (2018xj22zd), Anhui Province Education and Teaching Reform Commissioned Project (2018jyxm1463), and Anhui Jianzhu University Research Startup Project (2019QDZ61).

\section{References}

[1] S. Zheng, X. Tang, B. Song, S. Lu, and B. Ye, "Stable adaptive pi control for permanent magnet synchronous motor drive based on improved jitl technique," ISA Transactions, vol. 52, no. 4, pp. 539-549, 2013. 
[2] S. Zheng, X. Tang, and B. Song, "A graphical tuning method of fractional order proportional integral derivative controllers for interval fractional order plant," Journal of Process Control, vol. 24, no. 11, pp. 1691-1709, 2014.

[3] J. C. Thrash, K. W. Seitz, B. J. Baker et al., "Metabolic roles of uncultivated bacterioplankton lineages in the northern gulf of Mexico dead zone," Mbio, vol. 8, no. 5, Article ID e01017, 2017.

[4] W. Shi, R. Luo, and B. Li, "Adaptive fuzzy prescribed performance control for mimo nonlinear systems with unknown control direction and unknown dead-zone inputs," ISA Transactions, vol. 66, pp. 86-95, 2017.

[5] Z. Regály, A. Juhász, and D. Nehéz, "Interpreting brightness asymmetries in transition disks: vortex at dead zone or planetcarved gap edges?," The Astrophysical Journal, vol. 851, no. 2, p. 89, 2017.

[6] Q. Chen, X. Ren, J. Na, and D. Zheng, "Adaptive robust finitetime neural control of uncertain pmsm servo system with nonlinear dead zone," Neural Computing and Applications, vol. 28, no. 12, pp. 3725-3736, 2017.

[7] Y.-J. Liu and S. Tong, "Adaptive nn tracking control of uncertain nonlinear discrete-time systems with nonaffine deadzone input," IEEE Transactions on Cybernetics, vol. 45, no. 3, pp. 497-505, 2014.

[8] Z. Li, Z. Chen, J. Fu, and C. Sun, "Direct adaptive controller for uncertain mimo dynamic systems with time-varying delay and dead-zone inputs," Automatica, vol. 63, pp. 287-291, 2016.

[9] H. Ma, H. Liang, H. Ma, and Q. Zhou, "Nussbaum gain adaptive backstepping control of nonlinear strict-feedback systems with unmodeled dynamics and unknown dead zone," International Journal of Robust and Nonlinear Control, vol. 28, no. 17, pp. 5326-5343, 2018.

[10] Y.-J. Liu, Y. Gao, S. Tong, and C. P. Chen, “A unified approach to adaptive neural control for nonlinear discrete-time systems with nonlinear dead-zone input," IEEE Transactions on Neural Networks and Learning Systems, vol. 27, no. 1, pp. 139-150, 2015.

[11] Y. Li, S. Tong, and T. Li, "Observer-based adaptive fuzzy tracking control of mimo stochastic nonlinear systems with unknown control directions and unknown dead zones," IEEE Transactions on Fuzzy Systems, vol. 23, no. 4, pp. 1228-1241, 2014.

[12] B. Xu, "Robust adaptive neural control of flexible hypersonic flight vehicle with dead-zone input nonlinearity," Nonlinear Dynamics, vol. 80, no. 3, pp. 1509-1520, 2015.

[13] Y. Wang and J. Hu, "Improved prescribed performance control for air-breathing hypersonic vehicles with unknown deadzone input nonlinearity," ISA Transactions, vol. 79, pp. 95-107, 2018

[14] Q. Yang and M. Chen, "Adaptive neural prescribed performance tracking control for near space vehicles with input nonlinearity," Neurocomputing, vol. 174, pp. 780-789, 2016.

[15] J. Wei, Y. Zhang, M. Sun, and B. Geng, "Adaptive neural synchronization control of chaotic systems with unknown control directions under input saturation," Optik, vol. 132, pp. 249-261, 2017.

[16] J. Cao, G. Stamov, I. Stamova, and S. Simeonov, “Almost periodicity in impulsive fractional-order reaction-diffusion neural networks with time-varying delays," IEEE Transactions on Cybernetics, vol. 20, 2020.

[17] C. P. Bechlioulis and G. A. Rovithakis, "Robust adaptive control of feedback linearizable mimo nonlinear systems with prescribed performance," IEEE Transactions on Automatic Control, vol. 53, no. 9, pp. 2090-2099, 2008.
[18] C. P. Bechlioulis and G. A. Rovithakis, "Robust partial-state feedback prescribed performance control of cascade systems with unknown nonlinearities," IEEE Transactions on Automatic Control, vol. 56, no. 9, pp. 2224-2230, 2011.

[19] Y. Li, S. Tong, L. Liu, and G. Feng, "Adaptive output-feedback control design with prescribed performance for switched nonlinear systems," Automatica, vol. 80, pp. 225-231, 2017.

[20] C.-C. Wang and G.-H. Yang, "Observer-based adaptive prescribed performance tracking control for nonlinear systems with unknown control direction and input saturation," Neurocomputing, vol. 284, pp. 17-26, 2018.

[21] Y. Pan and H. Yu, "Biomimetic hybrid feedback feedforward neural-network learning control," IEEE Transactions on Neural Networks and Learning Systems, vol. 28, no. 6, pp. 1481-1487, 2016.

[22] H. Liu, Y. Pan, S. Li, and Y. Chen, "Adaptive fuzzy backstepping control of fractional-order nonlinear systems," IEEE Transactions on Systems, Man, and Cybernetics: Systems, vol. 47, no. 8, pp. 2209-2217, 2017.

[23] Y. Pan, C. Yang, L. Pan, and H. Yu, "Integral sliding mode control: performance, modification, and improvement," IEEE Transactions on Industrial Informatics, vol. 14, no. 7, pp. 3087-3096, 2017.

[24] H. Liu, S. Li, H. Wang, and Y. Sun, "Adaptive fuzzy control for a class of unknown fractional-order neural networks subject to input nonlinearities and dead-zones," Information Sciences, vol. 454-455, pp. 30-45, 2018.

[25] A. Boulkroune, M. M'saad, and M. Farza, "Adaptive fuzzy system-based variable-structure controller for multivariable nonaffine nonlinear uncertain systems subject to actuator nonlinearities," Neural Computing and Applications, vol. 28, no. 11, pp. 3371-3384, 2017.

[26] Y. Zhou, H. Wang, and H. Liu, "Generalized function projective synchronization of incommensurate fractional-order chaotic systems with inputs saturation," International Journal of Fuzzy Systems, vol. 22, pp. 1-14, 2018.

[27] Y. Liu, J. H. Park, B.-Z. Guo, and Y. Shu, "Further results on stabilization of chaotic systems based on fuzzy memory sampled-data control," IEEE Transactions on Fuzzy Systems, vol. 26, no. 2, pp. 1040-1045, 2017.

[28] H. Li, L. Bai, Q. Zhou, R. Lu, and L. Wang, "Adaptive fuzzy control of stochastic nonstrict-feedback nonlinear systems with input saturation," IEEE Transactions on Systems, Man, and Cybernetics: Systems, vol. 47, no. 8, pp. 2185-2197, 2017.

[29] A. Boulkroune, L. Merazka, and H. Li, "Fuzzy adaptive statefeedback control scheme of uncertain nonlinear multivariable systems," IEEE Transactions on Fuzzy Systems, vol. 99, 2018.

[30] H. Li, J. Wang, H. Du, and H. R. Karimi, "Adaptive sliding mode control for takagi-sugeno fuzzy systems and its applications," IEEE Transactions on Fuzzy Systems, vol. 26, no. 2, pp. 531-542, 2017.

[31] H. Liu, S.-G. Li, H.-X. Wang, and G.-J. Li, "Adaptive fuzzy synchronization for a class of fractional-order neural networks," Chinese Physics B, vol. 26, no. 3, Article ID 030504, 2017.

[32] H. Liu, Y. Pan, and J. Cao, "Composite learning adaptive dynamic surface control of fractional-order nonlinear systems," IEEE Transactions on Cybernetics, vol. 50, no. 6, pp. 2557-2567, 2019. 